Accepted after revision: March 19, 2010

\title{
Microarray Analysis of Irradiated Growth Plate Zones following Laser Microdissection Shows Later Importance of Differentially Expressed Genes during Radiorecovery
}

\author{
Meredith R. Pritchard Jason A. Horton Lihini S. Keenawinna
}

Timothy A. Damron

SUNY Upstate Medical University, Syracuse, N.Y., USA

\section{Key Words}

Growth plate $\cdot$ Microarray $\cdot$ Chondrocytes $\cdot$ Irradiation $\cdot$ Rat

\begin{abstract}
Purpose: Potential targets for selective radiorecovery modulation were investigated via the identification of late upregulated genes and pathways during growth plate chondrocyte recovery. Methods and Materials: Three groups of six 5-week-old male Sprague-Dawley rats underwent fractionated irradiation to the right tibiae over 5 days totaling 17.5 Gy and were then killed at 7, 11, and 16 days following the first radiotherapy fraction. The growth plates were collected from the proximal tibiae bilaterally and subsequently underwent laser microdissection to separate reserve, perichondral, proliferative, and hypertrophic zones. Differential gene expression was analyzed between irradiated right and nonirradiated left tibiae using RAE230 2.0 GeneChip microarray, compared between zones and time points, and subjected to functional pathway cluster analysis with real-time polymerase chain reaction (PCR) to confirm selected results. Results: The reserve zone showed the greatest number of differentially expressed genes and enriched pathways: 259 and 134, respectively. Differentially expressed genes included: Timp3, Gpx1, Gas6, Notch2, VEGF, and HIF-1. Enriched pathways included the developmental processes of regeneration, antiapoptosis, developmental growth, tissue regen-
\end{abstract}

eration, mesenchymal cell proliferation, negative regulation of immune response, and determination of symmetry. The reserve zone late upregulation of genes was validated using real-time PCR for Mgp, Gas6, and Eef1a1. Conclusions: A significant difference in late upregulated genes between

\begin{tabular}{ll}
\hline Abbreviations used in this paper \\
\hline BCMSD & bone, cartilage, matrix, and/or skeletal development \\
Bnip3 & $\begin{array}{l}\text { BCL2/adenovirus E1B 19-kDa-interacting protein 3 } \\
\text { confidence level }\end{array}$ \\
EL & extracellular matrix \\
FEM & fold enrichment score \\
GEO & Gene Expression Omnibus \\
GO & Gene Ontology \\
HIF-1 & hypoxia-indicible factor-1 \\
HZ & hypertrophic zone \\
IUCAC & Institutional Use and Care of Animals Committee \\
LMD & laser microdissection \\
Lox & lysyl oxidase \\
PC & perichondrium \\
PCR & polymerase chain reaction \\
PZ & proliferative zone \\
RMA & robust multiarray analysis \\
RZ & reserve zone \\
SD & Sprague-Dawley \\
SOM & self-organizing maps \\
VEGF & vascular endothelial growth factor \\
\hline
\end{tabular}

\section{KARGER}

(c) 2010 S. Karger AG, Basel

Fax +4161306 1234 E-Mail karger@karger.ch www.karger.com www.karger.com/cto
Dr. Timothy A. Damron

Department of Orthopedic Surgery

SUNY Upstate Medical University

6620 Fly Road, Suite 100 \& 200, East Syracuse, NY 13057 (USA)

Tel. +1 315464 8654, Fax +1 315464 6446, E-Mail damront@ upstate.edu 
growth plate zones exists. The reserve zone shows the greatest change, containing a 10 -fold increase in the total number of genes differentially expressed between days 7 and 16 . These findings suggest that reserve zone chondrocytes may play a later role in growth plate recovery response following irradiation.

Copyright $\odot 2010$ S. Karger AG, Basel
(16 days) differential upregulation of specific gene expression exists between irradiated and nonirradiated reserve zone (RZ), proliferative zone (PZ), perichondrium (PC), and hypertrophic zone (HZ) SD rat chondrocytes and that some factors potentially vital to growth plate recovery would follow the pattern of late upregulation.

\section{Introduction}

The longitudinal growth of bone is known to be a result of the orderly coordination of chondrocyte proliferation and hypertrophy, calcification of the matrix, vascular invasion, and completion of endochondral bone formation in the growth plate [Butler et al., 1990; Goldwein, 1991; Robertson et al., 1991]. Radiotherapy for bone or soft-tissue sarcomas close to the growth plate can result in the development of growth arrest, angular deformity, and/or limb length discrepancy. Potential for postirradiation recovery exists in the growth plate, based both on the variability of clinical outcomes following growth plate irradiation and on our own work in the weanling Sprague-Dawley (SD) rat model [Damron et al., 2000, 2004a].

Current analysis has focused on obtaining a better understanding of growth plate recovery in order to develop potential selective radiorecovery agents for clinical use during the radiotherapy treatment of pediatric solid tumors. Initial work in this animal model focused on evaluating the hypothesis that early (7-11 days after first radiation fraction) upregulated genes and pathways may be involved in growth plate recovery. However, an alternative explanation for early growth plate changes after irradiation is that they are reparative or 'injury responsive' rather than a true means of growth plate recovery. If the alternative explanation is true, later growth plate changes may actually lay the foundation for true recovery.

Hence, in this paper, we propose that key late (16 days after initial radiation) upregulated genes may provide an understanding of the mechanism of this recovery and suggest means for the development of novel radiorecovery agents. Furthermore, later upregulated genes may be the result of earlier changes in upstream mediators. This report utilized laser microdissection (LMD) and microarray to separate and characterize the gene expression of 4 zones of the SD rat growth plate after clinically relevant fractionated irradiation and to determine the enrichment of Gene Ontology (GO) functional groups involving significantly changed genes. Our hypothesis was that a late

\section{Methods}

All animal procedures were reviewed and approved by the Institutional Use and Care of Animals Committee (IUCAC). Three groups of six 5-week-old male SD rats underwent fractionated irradiation to the right tibiae over 5 days totaling $17.5 \mathrm{~Gy}$. The leftside tibiae served as nonirradiated controls. At 7, 11, and 16 days following the first radiotherapy fraction, 1 group of 6 animals each was killed by carbon dioxide asphyxiation, and the growth plates were collected from the proximal tibiae bilaterally, immediately frozen in liquid nitrogen, and stored at $-70^{\circ} \mathrm{C}$. The entire collecting process for each animal was completed within 2 min.

Time points were chosen based upon previous work showing that, from a histological perspective, the growth plate reaches its most disorganized, paucicellular state at approximately 1 week after irradiation. Gradual changes have been noted thereafter, with clones of regenerating chondrocytes clearly identified beginning at 2 weeks after irradiation but continuing to progress over time to a more fully recovered histological appearance and improved growth rate [Damron et al., 2008].

\section{Laser Microdissection}

Serial sections (6 $\mu \mathrm{m}$ for LMD) were cut on a Leica CM3050 cryostat immediately before the planned LMD. The LMD, RNA extraction and GeneChip Hybridization procedures were previously described [Wang et al., 2007]. For each of the 4 zones at each of the 3 time points, equal amounts of RNA from the 3 samples derived from 3 different limbs on each side (right and left) were pooled to create a single sample to reduce the individual variability and sampling errors, resulting in 8 samples of RNA from the 6 limbs at each time point, and 24 samples in all (online suppl. fig. 1, www.karger.com/doi/10.1159/000318644). The 24 RNA samples included the right and left $\mathrm{RZ}$ at 16 days, the right and left $\mathrm{PZ}$ at 16 days, the right and left $\mathrm{HZ}$ at 16 days, and the right and left PC at 16 days. Identical sets for 7 and 11 days were the focus of earlier reports [Damron et al., 2009; Zhang et al., 2008]. Each pooled sample had 30-50 ng RNA.

\section{Real-Time RT-Polymerase Chain Reaction}

Real-time quantitative RT-polymerase chain reaction (PCR) was performed as previously described. The $25-\mu \mathrm{l}$ reaction consisted of SYBR Green PCR Master Mix (PE Applied Biosystems, Carlsbad, Calif., USA), rat-specific primers for genes Mgp, growth arrest-specific gene 6 (Gas6), and Eeflal at a concentration of $300 \mathrm{nM}$, and $5 \mathrm{ng}$ pooled cDNA (table 1). All samples were run in triplicate along with $\beta$-actin as the reference gene (forward, 5'GGTCATAAGCTTGCGTTGAT3'; reverse, 5'TCAAGTTCGACCGTCTTCTC3') and no template controls. 
Table 1. $\log _{2}$ ratio of microarray and real-time PCR between irradiated and nonirradiated growth plate RZ

\begin{tabular}{|c|c|c|c|c|}
\hline \multirow[t]{2}{*}{ Probe ID } & \multirow[t]{2}{*}{ Gene title } & \multirow{2}{*}{$\begin{array}{l}\text { Gene } \\
\text { symbol }\end{array}$} & \multicolumn{2}{|l|}{16 days, $\mathrm{RZ}$} \\
\hline & & & $\begin{array}{l}\text { microarray } \\
\log _{2} \text { ratio }\end{array}$ & $\begin{array}{l}\text { real-time } \\
\text { PCR }\end{array}$ \\
\hline 1367568_a_at & matrix Gla protein & MGP & 4.13 & 2.26 \\
\hline 1383047_at & growth arrest-specific 6 & Gas6 & 4.04 & 1.92 \\
\hline 1370109_s_at & $\begin{array}{l}\text { eukaryotic translation } \\
\text { elongation factor } 1 \text { alpha } 1\end{array}$ & Eef1a1 & 3.70 & 6.98 \\
\hline
\end{tabular}

Analysis of Changes in Gene Expression

To identify differentially expressed genes in RZ, PZ, HZ, and PC, Affymetrix GCOS/MAS5 was used to calculate the intensity of the signal from each perfect-match probe relative to the signal for the mismatch probe, to determine whether or not the gene was present in the sample, and to generate a measure of the expression level of the gene for all zones [Wang et al., 2007]. Next, GeneSpring GX (Agilent Technologies, Palo Alto, Calif., USA) was used to identify differentially expressed genes in right tibial zonal samples which were normalized to the left tibia at each of the 3 time points using the robust multiarray analysis (RMA) method. An expression level filter of 10-fold change was then applied in GeneSpring to the normalized data. Meaningful differential expression was determined at the $99.9 \%$ confidence level (CL) [Damron et al., 2009]. We included genes at the extreme tails of the distribution curve, $\pm 3.3 \mathrm{z}$-scores from the mean, rather than by an arbitrary fold difference.

Two additional steps were used for the microarray analysis: clustering self-organizing maps (SOM) and pathway analysis. Gene lists were clustered by SOM in GeneSpring, and clusters fitting the hypothesized temporal expression pattern of interest (upregulation at 16 days after initial irradiation) were selected within each zone (RZ, PZ, HZ, and PC) for additional pathway analysis. Based on our hypothesis that the pattern of importance was limited to those genes that were significantly upregulated at 16 days on the irradiated side compared to the nonirradiated side, in most cases we used a $3 \times 2$ matrix for cluster analysis and selected clusters which fit the hypothesis and showed the highest degree of upregulation when more than 1 applicable cluster presented. Alternatively, for the RZ, with 259 genes passing the expression level filter, a $3 \times 2$ matrix did not adequately separate out a limited number of clusters which fit the desired pattern. Thus, we discarded the SOM and analyzed the genes of the RZ as one large group.

Functional pathway analysis (GO enrichment) of growth plate zone clusters $\mathrm{PZ}, \mathrm{PC}, \mathrm{HZ}$, and a total gene list as in the $\mathrm{RZ}$, was accomplished using hypergeometric $\mathrm{p}$ values to confirm significance, accepting hypergeometric $p$ values $<0.05$. In addition to the hypergeometric $\mathrm{p}$ values, pathways also had to include $>1$ probe set from the experimental data set and to have a fold enrichment score (FES) $\geq 5$ in order to be considered meaningful. For the $\mathrm{RZ}$, an additional filter requiring $\geq 5$ probe sets from the experimental data set and an FES $\geq 5$ was applied in order to create a list of the most concentrated enriched pathways with probe sets from the experimental data set. The additional enrichment filter was not presented in this paper since, when applied to the PZ, PC, and $\mathrm{HZ}$, it reduced the number of enriched pathways to 8,0 , and

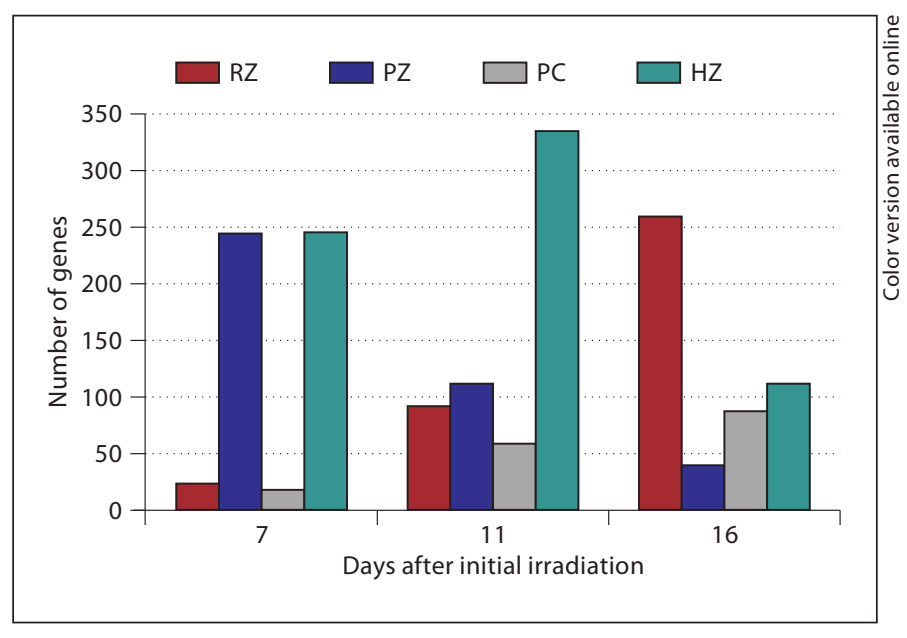

Fig. 1. Number of genes showing differential upregulation at 7, 11, and 16 days after initial irradiation of RZ, PZ, PC, and HZ.

0 , respectively. We also identified enriched GO groups that are involved in skeletal development and bone remodeling. The latter screen was determined based on a search utilizing AmiGO, a search engine for the GO database (http://amigo.geneontology. org/cgi-bin/amigo/go.cgi). The experimental series of data files has been deposited into the Gene Expression Omnibus (GEO) at the NCBI (accession GSE9537).

\section{Results}

Of the 31,099 probe sets arrayed on the chips, the temporal pattern of differential gene expression differed greatly between zones over time after the initiation of irradiation (fig. 1). Two hundred fifty-nine RZ genes, $40 \mathrm{PZ}$ genes, $111 \mathrm{HZ}$ genes, and $87 \mathrm{PC}$ genes having late (16 days after the initiation of the first radiation fraction) differential expression within the previously defined parameters were selected for further analysis.

Reserve Zone. For the RZ genes, the pathway analysis of all 259 genes passing the differential expression level 
Table 2. RZ: 38 differentially expressed genes which follow our hypothesized pattern included in the 16 enriched pathways

\begin{tabular}{|c|c|c|c|c|c|c|c|c|}
\hline \multirow[t]{2}{*}{ Probe ID } & \multirow[t]{2}{*}{ Gene title } & \multirow{2}{*}{$\begin{array}{l}\text { Gene } \\
\text { symbol }\end{array}$} & \multicolumn{3}{|c|}{$\log _{2}$ ratio $^{1}$} & \multicolumn{3}{|c|}{ Fold change ${ }^{2}$} \\
\hline & & & 7 days & 11 days & 16 days & 7 days & 11 days & 16 days \\
\hline 1389836_a_at & tissue inhibitor of metalloproteinase 3 & Timp3 & -1.39 & 1.38 & 2.83 & -2.62 & 2.60 & 7.11 \\
\hline 1367576_at & glutathione peroxidase 1 & Gpx1 & -2.05 & -0.95 & 2.84 & -4.15 & -1.93 & 7.14 \\
\hline 1370959_at & procollagen, type III, alpha 1 & Col3al & -2.78 & -0.15 & 2.86 & -6.87 & -1.11 & 7.25 \\
\hline 1370301_at & matrix metallopeptidase 2 & Mmp2 & -1.97 & 1.74 & 4.00 & -3.92 & 3.33 & 16.04 \\
\hline 1370234_at & fibronectin 1 & Fn1 & -3.03 & 0.31 & 3.19 & -8.18 & 1.24 & 9.12 \\
\hline 1389891_at & procollagen, type XI, alpha 2 (mapped) & Coll1a2 & -3.60 & 1.42 & 3.15 & -12.14 & 2.68 & 8.90 \\
\hline 1367586_at & lactate dehydrogenase A & Ldha & -1.38 & 0.82 & 2.60 & -2.61 & 1.76 & 6.06 \\
\hline 1388544_at & 2,3-bisphosphoglycerate mutase & Bpgm & -3.10 & -2.31 & 2.88 & -8.58 & -4.96 & 7.35 \\
\hline 1370341_at & enolase 2 , gamma & Eno2 & -3.14 & 0.26 & 4.41 & -8.83 & 1.20 & 21.22 \\
\hline 1372182_at & phosphofructokinase, platelet & Pfkp & -1.41 & -0.20 & 3.30 & -2.66 & -1.15 & 9.86 \\
\hline 1387361_s_at & phosphoglycerate kinase 1 & Pgk1 & -3.05 & 0.25 & 3.17 & -8.29 & 1.19 & 8.98 \\
\hline 1370927_at & procollagen, type XII, alpha 1 & Col12a1 & -3.24 & 0.85 & 3.95 & -9.46 & 1.80 & 15.42 \\
\hline 1373807_at & vascular endothelial growth factor $\mathrm{A}$ & Vegfa & -2.51 & 1.26 & 2.82 & -5.68 & 2.40 & 7.08 \\
\hline 1371412_a_at & neuronal regeneration-related protein & Nrep & -3.92 & 1.04 & 4.60 & -15.13 & 2.06 & 24.19 \\
\hline 1398362_at & Notch gene homolog 2 (Drosophila) & Notch2 & -2.70 & -0.38 & 2.85 & -6.50 & -1.30 & 7.22 \\
\hline 1387355_at & aggrecan 1 & Agcl & -3.78 & 1.45 & 2.64 & -13.71 & 2.73 & 6.22 \\
\hline 1368172_a_at & lysyl oxidase & Lox & -0.12 & -1.33 & 3.18 & -1.08 & -2.51 & 9.04 \\
\hline 1370944_at & procollagen, type $\mathrm{X}$, alpha 1 & Col10a1 & -3.04 & -0.79 & 5.47 & -8.25 & -1.73 & 44.36 \\
\hline 1383047_at & growth arrest-specific 6 & Gas6 & -2.53 & 1.31 & 4.04 & -5.76 & 2.47 & 16.49 \\
\hline 1367697_at & mitogen-activated protein kinase 14 & Mapk14 & -0.91 & -0.32 & 2.59 & -1.88 & -1.25 & 6.01 \\
\hline 1367563_at & secreted acidic cysteine-rich glycoprotein & Sparc & -6.16 & -0.58 & 4.19 & -71.36 & -1.49 & 18.24 \\
\hline 1388634_at & phosphoglucomutase 1 & Pgm1 & -1.30 & -0.29 & 2.88 & -2.46 & -1.22 & 7.37 \\
\hline 1387805_at & BCL2/adenovirus E1B 19-kDa-interacting protein 3 & Bnip3 & -0.24 & 0.93 & 3.47 & -1.18 & 1.91 & 11.10 \\
\hline 1381857_at & RAS p 21 protein activator 1 & Rasal & -1.66 & -1.15 & 2.49 & -3.15 & -2.22 & 5.61 \\
\hline 1387197_at & osteomodulin & Omd & -4.21 & 0.75 & 4.56 & -18.49 & 1.68 & 23.56 \\
\hline 1368788_at & chondroadherin & Chad & -3.31 & 0.15 & 4.58 & -9.90 & 1.11 & 23.88 \\
\hline 1367594_at & biglycan & Bgn & -3.57 & 1.18 & 4.24 & -11.86 & 2.26 & 18.95 \\
\hline 1392171_at & chitinase 3 -like 1 & Chi3l1 & -4.34 & -0.46 & 3.14 & -20.20 & -1.37 & 8.84 \\
\hline 1388311_at & Morf4 family-associated protein 1 & Mrfap1 & -2.54 & -0.98 & 2.55 & -5.80 & -1.98 & 5.86 \\
\hline 1387915_at & selenoprotein S & Sels & -2.46 & 0.35 & 3.16 & -5.52 & 1.27 & 8.96 \\
\hline 1370236_at & palmitoyl-protein thioesterase 1 & Ppt1 & -1.63 & -0.24 & 3.30 & -3.08 & -1.18 & 9.83 \\
\hline 1388416_at & low-density lipoprotein receptor-related protein 1 & Lrp1 & -3.58 & 0.66 & 3.94 & -11.92 & 1.58 & 15.34 \\
\hline 1389966_at & procollagen, type VI, alpha 3 (predicted) & Col6a3 & -2.77 & 0.63 & 3.00 & -6.84 & 1.55 & 7.98 \\
\hline 1370109_s_at & eukaryotic translation elongation factor 1 alpha 1 & Eefla1 & -2.43 & -1.03 & 3.70 & -5.40 & -2.04 & 13.03 \\
\hline 1370035_at & v-Ki-ras2 Kirsten rat sarcoma viral oncogene homolog & Kras & -2.00 & -1.51 & 3.03 & -4.01 & -2.85 & 8.15 \\
\hline 1370156_at & prion protein & Prnp & -2.58 & 1.87 & 2.80 & -5.97 & 3.65 & 6.96 \\
\hline 1372067_at & thioredoxin domain containing 1 & Txndc1 & -2.64 & -0.15 & 2.58 & -6.22 & -1.11 & 6.00 \\
\hline 1390846_at & procollagen, type XVI, alpha 1 & Col16a1 & -3.89 & 0.33 & 2.47 & -14.80 & 1.26 & 5.56 \\
\hline
\end{tabular}

filters (since all of the clusters followed the hypothesized pattern) showed 134 enriched pathways with a minimum of 2 probe sets and a minimum FES of 5 (online suppl. table 1). These pathways included 15 molecular, 21 cellular, and 98 biological pathways. Eight pathways (6\%) were related to bone, cartilage, matrix, and/or skeletal development (BCMSD). The additional filter for pathway analysis (minimum 5 probe sets in addition to an FES of 5) revealed just 16 enriched pathways (online suppl. table 2).
These pathways include 1 molecular, 3 cellular, and 12 biological pathways. Three of the pathways (19\%) were related to BCMSD. These 16 enriched pathways also revealed a more concentrated gene list of 38 differentially expressed genes (table 2).

Proliferative Zone. For the PZ genes, only 1 cluster (2, 1) from the $3 \times 2 \mathrm{SOM}$ followed the hypothesized pattern (online suppl. fig. 2). This cluster contained 9 genes that showed enrichment in $35 \mathrm{GO}$ terms with a minimum of 
Table 3. PZ: 9 differentially expressed genes which follow our hypothesized pattern

\begin{tabular}{|c|c|c|c|c|c|c|c|c|}
\hline \multirow[t]{2}{*}{ Probe ID } & \multirow[t]{2}{*}{ Gene title } & \multirow{2}{*}{$\begin{array}{l}\text { Gene } \\
\text { symbol }\end{array}$} & \multicolumn{3}{|c|}{$\log _{2}$ ratio $^{1}$} & \multicolumn{3}{|c|}{ Fold change $^{2}$} \\
\hline & & & 7 days & 11 days & 16 days & 7 days & 11 days & 16 days \\
\hline 1368416_at & integrin-binding sialoprotein & Ibsp & 4.58 & -0.23 & 5.16 & 23.84 & -1.17 & 35.74 \\
\hline 1373463_at & procollagen, type $\mathrm{V}$, alpha 2 & Col5a2 & 0.67 & 1.44 & 4.28 & 1.59 & 2.72 & 19.37 \\
\hline 1388204_at & matrix metallopeptidase 13 & Mmp13 & 3.41 & -0.21 & 4.25 & 10.63 & -1.15 & 18.97 \\
\hline 1370864_at & procollagen, type 1 , alpha 1 & Colla1 & 2.73 & 1.38 & 3.75 & 6.65 & 2.60 & 13.48 \\
\hline 1387854_at & procollagen, type 1 , alpha 2 & Col1a2 & 1.54 & 0.38 & 2.88 & 2.91 & 1.30 & 7.38 \\
\hline 1367749_at & lumican & Lum & -2.24 & -0.09 & 1.86 & -4.72 & -1.07 & 3.62 \\
\hline 1392981_at & iroquois-related homeobox 4 & $\operatorname{Irx} 4$ & -1.25 & 0.72 & 1.38 & -2.38 & 1.64 & 2.61 \\
\hline 1376185_at & kinesin family member C1 & Kifc1 & 0.38 & 0.09 & 1.30 & 1.30 & 1.06 & 2.47 \\
\hline 1368223_at & $\begin{array}{l}\text { a disintegrin-like and metallopeptidase (reprolysin type) } \\
\text { with thrombospondin type } 1 \text { motif, } 1\end{array}$ & Adamts1 & 0.11 & -0.05 & 1.27 & 1.08 & -1.03 & 2.41 \\
\hline
\end{tabular}

${ }^{1}$ Red $=$ Falls within $+99.9 \%$ CL; green $=$ falls within $-99.9 \% \mathrm{CL}$

${ }^{2}$ Red $=$ Fold change $\geq+2$; green $=$ fold change $\leq-2$.

2 probe sets and a minimum FES of 5 (table 3). These pathways included 23 biological, 6 cellular, and 6 molecular pathways. Seven pathways (20\%) are involved in BCMSD (online suppl. table 3).

Perichondrium. For the PC genes, 2 clusters from the $3 \times 2$ SOM showed the trend of hypothesized important clusters $(1,1)$ and $(1,2)$ (online suppl. fig. 3 ). Cluster $(1,1)$ contained 20 genes and showed enrichment in 53 GO terms (table 4; online suppl. table 4). Five of the original 20 genes are involved in the 53 enriched GO terms which met our enrichment criteria. These pathways included 47 biological, 2 cellular, and 6 molecular pathways. None of these terms are involved in BCMSD, however.

Cluster $(1,2)$ contained 15 genes and showed enrichment in 5 GO terms (table 4; online suppl. fig. 4). These pathways included only 5 molecular pathways ( 0 biological and 0 cellular). None of these terms are involved in BCMSD either.

Hypertrophic Zone. For the HZ genes, cluster $(2,2)$ from the $3 \times 2$ SOM showed the temporal trend of hypothesized importance (online suppl. fig. 4). In pathway analysis of the 17 genes in this cluster, 18 pathways showed enrichment (table 5; online suppl. table 5). These pathways included only 18 biological pathways ( 0 cellular and 0 molecular). None of these terms are involved in BCMSD.

Overlapping and unique pathways related to BCMSD for each of the growth plate zones are shown in table 6 . Additionally, total differentially expressed genes for RZ, $\mathrm{PZ}, \mathrm{PC}$, and $\mathrm{HZ}$ at each of the 3 time points examined by microarray are shown in figure 1.

\section{Real-Time Results}

To confirm microarray results, real-time RT-PCR was performed with a set of rat-specific primers and template cDNA generated by RT-PCR. The primers were designed based on selected genes among the late upregulated $\mathrm{RZ}$ data set.

\section{Discussion}

The treatment for musculoskeletal oncology tumors in skeletally immature patients occasionally involves radiotherapy, which has a documented damaging effect on growth plate chondrocytes [Damron et al., 2000, 2009]. As a result, the beneficial effect of radioprotectants in selectively preventing damage to the growth plate has been explored. As yet, the chondroprotective effects of these drugs are incomplete. The use of selective radiorecovery agents to stimulate growth plate return without stimulating tumor growth has been explored in the past in our laboratory, and animal models have suggested that the irradiated growth plate has the potential to recover after injury [Damron et al., 2003, 2004b; Horton et al., 2006]. We hypothesize that some factors potentially vital to growth plate recovery would follow a pattern of late upregulation, specifically at 16 days following the initial irradiation fraction. In this experiment, laser microdissection was accomplished on 4 growth plate zones (RZ, PC, $\mathrm{PZ}$, and $\mathrm{HZ}$ ) following a clinically relevant fractionated $17.5-$ Gy $(5 \times 3.5$ Gy) irradiation. The purpose was to identify late upregulated genes which also may reveal novel upstream biological modulators that could be fu- 
Table 4. PC: differentially expressed genes which follow our hypothesized pattern for clusters $(1,1)$ and $(1,2)$

\begin{tabular}{|c|c|c|c|c|c|c|c|c|c|}
\hline \multirow{2}{*}{$\begin{array}{l}\text { GP } \\
\text { zone }\end{array}$} & \multirow[t]{2}{*}{ Probe ID } & \multirow[t]{2}{*}{ Gene title } & \multirow{2}{*}{$\begin{array}{l}\text { Gene } \\
\text { symbol }\end{array}$} & \multicolumn{3}{|c|}{$\log _{2}$ ratio $^{1}$} & \multicolumn{3}{|c|}{ Fold change $^{2}$} \\
\hline & & & & 7 days & 11 days & 16 days & 7 days & 11 days & 16 days \\
\hline \multirow{20}{*}{$\begin{array}{l}\text { PC } \\
\text { cluster } \\
(1,1)\end{array}$} & 1398275_at & matrix metallopeptidase 9 & Mmp9 & 0.12 & 0.38 & 4.70 & 1.09 & 1.31 & 26.07 \\
\hline & 1367948_a_at & kinase insert domain protein receptor & $\mathrm{Kdr}$ & 0.52 & 0.07 & 3.12 & 1.43 & 1.05 & 8.68 \\
\hline & 1373094_at & general transcription factor II $\mathrm{H}$, polypeptide 1 & Gtf2h1 & 1.04 & 0.16 & 2.54 & 2.05 & 1.12 & 5.80 \\
\hline & 1376914_at & DEP domain containing la & Depdcla & -0.47 & -0.28 & 2.43 & -1.38 & -1.21 & 5.38 \\
\hline & 1371245_a_at & similar to hemoglobin beta- 2 subunit & LOC689064 & -1.89 & -0.56 & 2.34 & -3.70 & -1.48 & 5.08 \\
\hline & 1373661_a_at & chemokine (C-X-C motif) receptor 4 & Cxcr4 & -1.50 & 0.57 & 2.23 & -2.82 & 1.49 & 4.68 \\
\hline & 1387134_at & schlafen 3 & Slfn3 & -0.32 & 0.28 & 2.13 & -1.25 & 1.21 & 4.37 \\
\hline & 1384449_at & E1A-binding protein p300 & Ep300 & -0.33 & -0.12 & 2.09 & -1.26 & -1.08 & 4.24 \\
\hline & 1368136_at & thymopoietin & Tmpo & -0.33 & -0.57 & 2.01 & -1.26 & -1.48 & 4.02 \\
\hline & 1377772_at & $\begin{array}{l}\text { transmembrane protein with EGF-like and two } \\
\text { follistatin-like domains } 1\end{array}$ & Tmeff1 & -1.02 & -1.45 & 2.01 & -2.03 & -2.73 & 4.01 \\
\hline & 1393458_s_at & PHD finger protein 14 & Phf14 & -1.08 & 0.17 & 1.98 & -2.11 & 1.13 & 3.95 \\
\hline & 1380513_at & RNA polymerase II-associated protein 2 & Rpap2 & 0.26 & 0.44 & 1.94 & 1.20 & 1.35 & 3.84 \\
\hline & 1380766_a_at & similar to RIKEN cDNA $8430427 \mathrm{H} 17$ gene & RGD1563510 & -0.45 & 0.01 & 1.87 & -1.37 & 1.01 & 3.66 \\
\hline & 1398455_at & zinc finger, DHHC domain containing 13 & Zdhhc13 & -0.11 & 0.44 & 1.84 & -1.08 & 1.36 & 3.57 \\
\hline & 1371774_at & spermidine/spermine N1-acetyl transferase & Sat1 & -0.47 & -0.54 & 1.82 & -1.39 & -1.46 & 3.52 \\
\hline & 1383641_at & endothelin receptor type $\mathrm{A}$ & Ednra & -0.83 & -0.79 & 1.73 & -1.77 & -1.73 & 3.31 \\
\hline & 1380596_at & transthyretin & $\operatorname{Ttr}$ & -0.10 & 0.29 & 1.70 & -1.07 & 1.22 & 3.24 \\
\hline & 1385660_at & SMEK homolog 1 , suppressor of mek1 & Smek1 & 0.10 & -0.21 & 1.68 & 1.07 & -1.15 & 3.21 \\
\hline & 1374422_at & similar to Erbb2 interacting protein isoform 2 & RGD1562952 & 0.31 & -1.33 & 1.62 & 1.24 & -2.52 & 3.08 \\
\hline & 1374253_at & similar to RIKEN cDNA $2310035 \mathrm{C} 23$ & RGD1307235 & -0.16 & -0.37 & 1.51 & -1.12 & -1.29 & 2.85 \\
\hline \multirow{15}{*}{$\begin{array}{l}\text { PC } \\
\text { cluster } \\
(1,2)\end{array}$} & 1389871_at & glutamate oxaloacetate transaminase 2 , mitochondrial & Got2 & -1.64 & -0.57 & 2.52 & -3.11 & -1.49 & 5.72 \\
\hline & 1377029_at & RAR-related orphan receptor alpha & Rora & 0.51 & -0.32 & 2.08 & 1.43 & -1.25 & 4.22 \\
\hline & 1392007_at & USP6 N-terminal like & Usp6nl & 0.38 & 0.33 & 1.99 & 1.30 & 1.26 & 3.97 \\
\hline & 1387005_at & cathepsin S & Ctss & -0.28 & 0.19 & 1.97 & -1.21 & 1.14 & 3.91 \\
\hline & 1376676_a_at & M-phase phosphoprotein 8 & Mphosph8 & 0.28 & 0.25 & 1.74 & 1.22 & 1.19 & 3.33 \\
\hline & 1372432_at & PRP3 pre-mRNA processing factor 3 homolog & Prpf3 & 1.17 & -0.60 & 1.72 & 2.26 & -1.52 & 3.30 \\
\hline & 1374273_at & Coxsackie virus and adenovirus receptor & Cxadr & 0.36 & -0.50 & 1.72 & 1.28 & -1.41 & 3.30 \\
\hline & 1386247_at & sorting nexin 25 & Snx25 & 0.21 & 0.17 & 1.72 & 1.16 & 1.13 & 3.29 \\
\hline & 1382419_at & centromere protein $\mathrm{K}$ & Cenpk & -0.47 & -1.37 & 1.71 & -1.38 & -2.59 & 3.27 \\
\hline & 1377903_at & similar to intersectin 2 ( $\mathrm{SH} 3$ domain-containing protein $1 \mathrm{~B})$ & LOC313934 & 0.00 & 0.22 & 1.68 & -1.00 & 1.16 & 3.21 \\
\hline & 1398663_at & zinc finger protein 61 & Zfp61 & 0.24 & 0.19 & 1.67 & 1.18 & 1.14 & 3.17 \\
\hline & 1388960_at & pyrophosphatase (inorganic) 1 & Ppal & 1.32 & 0.09 & 1.65 & 2.50 & 1.07 & 3.15 \\
\hline & 1388724_at & cell division cycle 40 homolog & Cdc40 & -1.96 & 0.36 & 1.55 & -3.88 & 1.28 & 2.94 \\
\hline & 1377616_at & family with sequence similarity 92 , member A1 & Fam92a1 & -0.17 & -0.17 & 1.52 & -1.13 & -1.13 & 2.87 \\
\hline & 1373554_at & splA/ryanodine receptor domain and SOCS box containing 1 & Spsb1 & -1.49 & 0.52 & 1.52 & -2.81 & 1.43 & 2.87 \\
\hline
\end{tabular}

${ }^{1}$ Red $=$ Falls within $+99.9 \%$ CL; green = falls within $-99.9 \%$ CL.

${ }^{2}$ Red $=$ Fold change $\geq+2$; green $=$ fold change $\leq-2$.

ture targets in activating radiorecovery. Our hypotheses that (1) temporal gene differential expression patterns differ between irradiated and nonirradiated RZ, PZ, PC, and $\mathrm{HZ}$ and (2) some genes and/or pathways potentially vital to growth plate recovery follow a pattern of late upregulation (16 days) after irradiation are both confirmed.

Unique temporal patterns of growth plate chondrocyte gene expression after fractionated irradiation have emerged. Among the 4 growth plate zones studied, the irradiated RZ shows the greatest increase of 10 -fold differentially expressed genes from 7 to 16 days after initial irradiation (fig. 1). Moreover, all of the clusters for the RZ fit the aforementioned hypothesized pattern, while only 1 cluster (of 6) each for the $\mathrm{PZ}$ and $\mathrm{HZ}$, and 2 clusters (of 6) for the PC followed the proposed pattern. The RZ revealed the greatest number of genes (259) showing late upregulation, and consequently the RZ showed enrichment in the most functional pathways involving BCMSD (8 GO terms).

There were only 2 zones (RZ and PZ) showing late (16 days) enrichment of BCMSD ontologies in the growth plate, with 8 and 7 enriched pathways, respectively. These enriched pathways showed overlap in 3 ontologies between the 2 zones: extracellular matrix (ECM), ECM part, and ECM structural constituent. None of the enriched ontologies within the $\mathrm{PC}$ and the $\mathrm{HZ}$ were involved in BCMSD. 
Table 5. HZ: 17 differentially expressed genes from cluster $(2,2)$ which follow our hypothesized pattern

\begin{tabular}{|c|c|c|c|c|c|c|c|c|}
\hline \multirow[t]{2}{*}{ Probe ID } & \multirow[t]{2}{*}{ Gene title } & \multirow{2}{*}{$\begin{array}{l}\text { Gene } \\
\text { symbol }\end{array}$} & \multicolumn{3}{|c|}{$\log _{2}$ ratio $^{1}$} & \multicolumn{3}{|c|}{ Fold change ${ }^{2}$} \\
\hline & & & 7 days & 11 days & 16 days & 7 days & 11 days & 16 days \\
\hline 1387125_at & S100 calcium-binding protein A9 (calgranulin B) & S100a9 & -2.19 & -0.85 & 4.21 & -4.56 & -1.80 & 18.54 \\
\hline 1368494_at & S100 calcium-binding protein A8 (calgranulin A) & S100a8 & -3.79 & -0.06 & 4.13 & -13.80 & -1.05 & 17.57 \\
\hline 1370913_at & radical S-adenosyl methionine domain containing 2 & Rsad2 & -0.82 & 0.19 & 3.67 & -1.76 & 1.14 & 12.70 \\
\hline 1380366_at & similar to immunoglobulin heavy chain 6 (Igh-6) & RGD1359202 & -0.65 & 0.04 & 3.64 & -1.57 & 1.03 & 12.46 \\
\hline 1390388_at & ferrochelatase & Fech & -0.34 & -0.43 & 3.55 & -1.26 & -1.35 & 11.71 \\
\hline 1368477_at & ATPase, $\mathrm{Ca}^{2+}$-transporting, ubiquitous & Atp2a3 & 0.28 & 0.01 & 3.33 & 1.21 & 1.00 & 10.06 \\
\hline 1373881_at & rho, GDP dissociation inhibitor (GDI) beta & Arhgdib & 0.34 & 0.56 & 2.88 & 1.27 & 1.47 & 7.35 \\
\hline 1377998_at & coproporphyrinogen oxidase & Cpox & -1.64 & 0.88 & 2.86 & -3.11 & 1.84 & 7.25 \\
\hline 1387921_at & zinc finger $\mathrm{CCCH}$ type containing 14 & Zc3h14 & 0.86 & 0.00 & 2.84 & 1.81 & 1.00 & 7.16 \\
\hline 1371953_at & cyclin G2 & Ccng2 & 0.47 & 0.31 & 2.77 & 1.38 & 1.24 & 6.82 \\
\hline 1386901_at & cd36 antigen & $\mathrm{Cd} 36$ & 0.51 & -1.92 & 2.55 & 1.43 & -3.77 & 5.85 \\
\hline 1379235_x_at & similar to CDC45L & Cdc45l & 0.72 & -0.12 & 2.53 & 1.65 & -1.09 & 5.77 \\
\hline 1373036_at & similar to Ras GTPase-activating-like protein IQGAP2 & RGD1561455 & 0.07 & -0.07 & 2.32 & 1.05 & -1.05 & 4.99 \\
\hline 1375414_at & $\begin{array}{l}\text { TAF9 RNA polymerase II, TATA box-binding protein } \\
\text { (TBP)-associated factor }\end{array}$ & Taf9 & 0.27 & -0.20 & 2.00 & 1.21 & -1.15 & 3.99 \\
\hline 1379335_at & polyadenylate-binding protein-interacting protein 1 & Paip1 & 0.41 & 0.29 & 1.90 & 1.33 & 1.23 & 3.73 \\
\hline 1368742_at & complement component 5 , receptor 1 & C5r1 & -1.77 & 0.85 & 1.79 & -3.42 & 1.81 & 3.45 \\
\hline 1398607_at & similar to KIAA0240 & RGD1305680 & 0.43 & -0.07 & 1.77 & 1.35 & -1.05 & 3.41 \\
\hline
\end{tabular}

${ }^{1}$ Red $=$ Falls within $+99.9 \%$ CL; green $=$ falls within $-99.9 \%$ CL.

${ }^{2}$ Red $=$ Fold change $\geq+2$; green $=$ fold change $\leq-2$.

\section{Enriched GO Terms and Genes Unique to $R Z$}

The RZ, the thinnest and least cellular zone of the GP, showed the greatest number of genes upregulated at 16 days after the initial radiation treatment. Although many of the upregulated RZ genes involved ECM GO terms, there were a few genes involving key developmental pathways. Previous microarray studies in our lab have suggested the importance of the ECM genes in all zones of the GP following irradiation. The ECM genes Sparc, Gas6, Fn1, Timp3, and Mmp2 and various procollagens have shown high expression levels in the RZ and PC following fractionated irradiation and have shown differential expression in irradiated compared to nonirradiated $\mathrm{PZ}$ and $\mathrm{HZ}$ following single-fraction irradiation [Zhang et al., 2007, 2008]. The differential expression of these related genes within the RZ in the current data set further supports their importance in radiorecovery. In this paper, the $\mathrm{RZ}$, when compared to the $\mathrm{PZ}, \mathrm{PC}$, and $\mathrm{HZ}$, also expresses a unique collection of genes from the developmental processes, many of which are involved in antiapoptosis (GO: 0006916) and wound healing (GO: 0042060).

Differentially expressed genes unique to the RZ data set include: Timp3, glutathione peroxidase 1 (Gpx1), Gas6, Notch2, VEGF, HIF-1, and Eefla1. The overexpression of Timp3 has been associated with antiangiogenic activity, a reduction in cell migration and invasion, and the initiation of apoptosis [Baker et al., 2002]. Gpx1 is a cellular antioxidant enzyme which is involved in neutralizing hydrogen peroxide similar to SOD enzymes [Koedam et al., 2002]. We have previously reported the presence of Gas6 in the growth plate, although its role there has not been established [Wang et al., 2007]. Its involvement in promoting differentiation into mature chondrocytes and calcification during late chondrogenesis may be similar to that of osteocalcin and MGP [Motomura et al., 2007]. Notch2 is a positive regulator of chondroprogenitor differentiation and proliferation that, when upregulated, may be a factor in the formation of regenerative chondrocyte clones through progenitor/RZ cell expansion and proliferation [Hardingham et al., 2006].

Another essential gene to the GP showing late upregulation in the RZ is vascular endothelial growth factor (VEGF), which plays a role in the vascular invasion of the growth plate and resultant apoptosis and bone formation [Koedam et al., 2002]. At low oxygen levels, the key mediator of an increased VEGF expression is hypoxia-inducible factor-1 (HIF-1) [Cramer et al., 2004]. VEGF expression is thought to be crucial in the coordination of chondrocyte death and ECM remodeling [Cramer et al., 2004]. Lysyl oxidase (Lox) and BCL2/adenovirus E1B 19-kDa-in- 
Table 6. BCMSD ontologies with upregulation at 16 days after initial irradiation

\begin{tabular}{|c|c|c|c|c|c|c|}
\hline \multirow{2}{*}{$\begin{array}{l}\text { GO parent } \\
\text { category }\end{array}$} & \multirow{2}{*}{$\begin{array}{l}\text { GO parent term } \\
\text { biological regulation/multicellular organismal process }\end{array}$} & \multirow{4}{*}{$\begin{array}{l}\text { GO (child) term } \\
\text { regulation of tissue remodeling } \\
\text { positive regulation of tissue remodeling } \\
\text { regulation of bone remodeling } \\
\text { positive regulation of bone remodeling }\end{array}$} & \multirow{2}{*}{$\begin{array}{l}\text { GO ID } \\
34103 \\
34105\end{array}$} & \multicolumn{3}{|c|}{ GP zone } \\
\hline & & & & $\mathrm{RZ}$ & $\mathrm{PZ}$ & $\begin{array}{l}\mathrm{HZ} \\
\mathrm{HZ}\end{array}$ \\
\hline & & & 46850 & \multirow[t]{2}{*}{$\mathrm{RZ}$} & \multirow[t]{2}{*}{$\mathrm{PZ}$} & $\mathrm{HZ}$ \\
\hline & & & 46852 & & & $\mathrm{HZ}$ \\
\hline & $\begin{array}{l}\text { cellular process/biological adhesion/multicellular } \\
\text { organismal process/developmental process }\end{array}$ & cartilage condensation & 1502 & \multicolumn{3}{|l|}{$\mathrm{RZ}$} \\
\hline & \multirow[t]{5}{*}{ developmental process/multicellular process } & tissue development & 9888 & \multirow{4}{*}{$\mathrm{RZ}$} & $\mathrm{PZ}$ & $\mathrm{HZ}$ \\
\hline & & bone mineralization & 30282 & & $\mathrm{PZ}$ & $\mathrm{HZ}$ \\
\hline & & biomineral formation & 31214 & & $\mathrm{PZ}$ & $\mathrm{HZ}$ \\
\hline & & regulation of ossification & 30278 & & $\mathrm{PZ}$ & $\mathrm{HZ}$ \\
\hline & & regulation of bone mineralization & 30500 & $\mathrm{RZ}$ & $\mathrm{PZ}$ & $\mathrm{HZ}$ \\
\hline & \multirow[t]{2}{*}{ multicellular organismal process } & bone remodeling & 46849 & & $\mathrm{PZ}$ & $\mathrm{HZ}$ \\
\hline & & tissue remodeling & 48771 & & $\mathrm{PZ}$ & $\mathrm{HZ}$ \\
\hline & multicellular organismal process/biological regulation & bone resorption & 45453 & \multicolumn{3}{|l|}{$\mathrm{RZ}$} \\
\hline & \multirow[t]{3}{*}{ multicellular organismal process/developmental process } & skeletal development & 1501 & & $\mathrm{PZ}$ & $\mathrm{HZ}$ \\
\hline & & ossification & 1503 & & $\mathrm{PZ}$ & $\mathrm{HZ}$ \\
\hline & & cartilage development & 51216 & $\mathrm{RZ}$ & $\mathrm{PZ}$ & $\mathrm{HZ}$ \\
\hline \multirow{2}{*}{$\begin{array}{l}\text { Cellular } \\
\text { component }\end{array}$} & \multirow[t]{2}{*}{ extracellular region } & extracellular matrix & 31012 & $\mathrm{RZ}$ & \multirow{2}{*}{\multicolumn{2}{|c|}{$\begin{array}{l}\mathrm{PZ} \\
\mathrm{PZ}\end{array}$}} \\
\hline & & extracellular matrix part & 44420 & $\mathrm{RZ}$ & & \\
\hline $\begin{array}{l}\text { Molecular } \\
\text { function }\end{array}$ & structural molecule activity & extracellular matrix structural constituent & 5201 & $\mathrm{RZ}$ & \multicolumn{2}{|l|}{$\mathrm{PZ}$} \\
\hline
\end{tabular}

None of the BCMSD GO terms are found in PC.

teracting protein 3 (Bnip3) are also regulated by Hif-1. An increased expression of Lox has been revealed in previous microarray studies in hypoxic tumor cells and is necessary for hypoxia-induced metastasis [Erler et al., 2006]. Also, transcription of Bnip3 has been shown to be significantly induced in response to hypoxia [Bruick, 2000]. Lox and Bnip3 both show differential expression and late upregulation with $\log _{2}$ ratios of 3.18 and 3.47 , respectively, at 16 days after initial irradiation (table 3). In our current study, Hif1 is upregulated in the $\mathrm{RZ}$ at 16 days after initial irradiation, albeit just below our differential expression cutoff values. Previous in vivo studies have shown that the growth plate is hypoxic and cell growth and survival is vitally dependent on Hif-1 [Cramer et al., 2004]. Further inspection of the genes involved in the enriched pathways via the GO database shows that: Vegf and Lox are included in the gene lists for ECM (GO ID: 31012), ECM part (GO ID: 44420), and proteinaceous ECM (GO ID: 5578); Vegf and Bnip3 are involved in antiapoptosis (GO ID: 6916), and Lox is involved in wound healing (GO ID: 42060). The upregulation of these genes in the RZ following irradiation suggests the possible activation of a VEGF-Hif-1 pathway in response to injury in the GP.

The upregulated gene Eefla1 has been speculated to be a promising marker in the detection of cellular senescence, which may reveal cellular status with long-term cellular responses to tumor treatment; recent studies show that in vivo senescence participates in tumor suppression [Byun et al., 2009].

\section{Enriched GO Terms Unique to the PZ}

The $\mathrm{PZ}$ provides a single cluster of upregulated ECMinvolved genes. As mentioned previously, earlier microarray studies in our lab have suggested the importance of the ECM genes in all zones of the GP following irradiation. The ECM genes Mmp13, Ibsp, Colla2, Lum, and Col5a2 have shown differential expression in irradiated compared to nonirradiated $\mathrm{PZ}$ and $\mathrm{HZ}$ following single fraction irradiation [Zhang et al., 2007]. A single gene not previously described in the growth plate to our knowledge, Irx4, was also upregulated in a differential fashion in the PZ. Irx4 is associated with just 2 GO terms: estab- 
lishment of organ orientation (GO: 0048561) and heart development (GO: 0007507). The GO term for establishment of organ orientation is downstream of our enriched GO term anatomical structure development (GO: 0048856), defined as the biological process whose specific outcome is the progression of an anatomical structure from an initial condition to its mature state. Since our earlier work has shown premature differentiation in PZ chondrocytes, the late upregulation of Irx4 may be involved in initializing the completion of proliferation in the GP after irradiation [Zhang et al., 2007].

\section{Differentially Expressed Genes Unique to $\mathrm{HZ}$}

Analysis of the HZ provided a single cluster following our hypothesized pattern. The genes from our data set present in these pathways include: ferrochelatase (Fech), complement component 5a receptor 1 (C5r1), S100 calcium-binding protein A9 (s100a9), S100 calcium-binding protein A8 (s100a8), and coproporphyrinogen oxidase (Cpox). Cpox and Fech are known enzymes of the heme biosynthetic pathway, specifically the 6th and terminal enzymes, respectively [Voet et al., 2002]. The resultant upregulation of these 2 genes may be due to a change in mitochondrial respiration and the invasion of surrounding metaphyseal blood vessels, mesenchymal cells, and osteoclasts [Abad et al., 1999]. s100a8 and s100a9 combine in the protein form as calprotectin, a calcium- and zinc-binding protein complex and an endogenous factor which is present in bodily fluids inducing apoptosis by the exclusion of zinc from target cells [Yui et al., 2002]. These 2 genes are significantly downregulated at 7 days following initial irradiation, then significantly upregulated at 16 days. C5r1 is a member of the chemoattractant receptor family which binds $\mathrm{C} 5 \mathrm{a}$ and releases calcium from intracellular stores. Upregulation of C5rl expression varies by tissue and is induced by prostaglandin $\mathrm{E} 2$, IL-6, TNF- $\alpha$, and IFN- $\gamma$ [Monk et al., 2007]. While IL-6, TNF- $\alpha$, and IFN- $\gamma$ do not show differential expression in this data set, prostaglandin $\mathrm{E}_{2}$ shows differential expression within our $99.9 \% \mathrm{CL}$ in the $\mathrm{HZ}$ with $\log _{2}$ ratio values of 2.07 and 2.04 at 11 and 16 days after initial fractionated irradiation, respectively.

It has been suggested that radical S-adenosyl methionine domain containing 2 (Rsad2) or Best5 play an important role in osteoblast function, and that one may play a role in the response of osteoblasts to stimuli that modulate proliferation/differentiation [Grewal et al., 2000]. Best 5 shows differential expression within our $99.9 \% \mathrm{CL}$ in the $\mathrm{HZ}$ with a $\log _{2}$ ratio of 3.67 at 16 days after initial fractionated irradiation.

\section{Differentially Expressed Genes and GO Terms Unique} to $P C$

Analysis of the PC resulted in 2 clusters falling within our hypothesized pattern. Pathway analysis of cluster (1, 1) reveals mostly vascular-related GO terms while cluster $(1,2)$ included enzyme- and lipid-binding GO terms. Cluster $(1,1)$ included the most interesting and previously researched group of genes, i.e. $\mathrm{Mmp} 9$, kinase insert domain protein receptor (KDR), chemokine (C-X-C motif) receptor 4 (Cxcr4), Tmpo, and endothelin receptor type A (Ednra), while cluster $(1,2)$ included a group of less studied genes, i.e. Got2, Prpf3, Snx25, and Ppa1. Ednra has been shown to bind to endothelin 1 and induce an increase in intracellular calcium [Kitten et al., 1997]. Cxcr4 is the known receptor to stromal cell-derived factor-1 (SDF-1); past arthritic studies have shown that the interaction between SDF-1- and Cxcr4-positive chondrocytes results in the elevation of MMP-3, a cartilage matrix-degrading enzyme [Kanbe et al., 2002]. KDR signaling has been shown to result in endothelial nitric oxide synthase upregulation and activation [Gille et al., 2001]. It has been found to be expressed during in vitro chondrocyte differentiation. Previous studies have revealed that blocking KDR resulted in the inhibition of VEGF, supporting the concept that the migration and invasion of endothelial cells are regulated by VEGF produced by chondrocytes via a paracrine angiogenic loop [Carlevaro et al., 2000]. Additionally, VEGF promotes MMP-9 secretion. Both VEGF and MMP-9 are key regulators in the remodeling of skeletal tissue, as well as in the recruitment and differentiation of endothelial cells, osteoclasts, chondrocytes, and osteoprogenitors [Ortega et al., 2003; Hall et al., 2006].

In summary, our current analysis has provided a select set of enriched functional pathways and genes for each zone of the growth plate. The RZ shows 10 times as many differentially expressed genes at the 16-day time point compared to 7 days. A thorough analysis of the specific genes and pathways has established that the RZ is important in the later recovery of the growth plate following irradiation. Further investigation is warranted to determine more specific biochemical signaling pathways involved in radiorecovery, their upstream biological modulators, and potential means for selective manipulation to achieve desirable selective radiorecovery responses.

\section{Acknowledgement}

This study was supported by NIH/NCI 2R01CA083892. 


\section{References}

-Abad, V., J.A. Uyeda, H.T. Temple, F. De Luca, J. Baron (1999) Determinants of spatial polarity in the growth plate. Endocrinology 140: 958-962.

Baker, A.H., D.R. Edwards, G. Murphy (2002) Metalloproteinase inhibitors: biological actions and therapeutic opportunities. J Cell Sci 115: 3719-3727.

Bruick, R.K. (2000) Expression of the gene encoding the proapoptotic Nip3 protein is induced by hypoxia. Proc Natl Acad Sci USA 97: 9082-9087.

Butler, M.S., W.W. Robertson, Jr., W. Rate, et al. (1990) Skeletal sequelae of radiation therapy for malignant childhood tumors. Clin Orthop Relat Res 251: 235-240.

Byun, H., N. Han, H. Lee, K. Kim, Y. Ko, G. Yoon, Y. Lee, S. Hong, J. Lee (2009) Cathepsin D and eukatyotic translation elongation factor 1 as promising markers of cellular senescence. Cancer Res 69: 4638-4647.

-Carlevaro, M.F., S. Cermelli, R. Cancedda, F.D. Cancedda (2000) Vascular endothelial growth factor (VEGF) in cartilage neovascularization and chondrocyte differentiation: auto-paracrine role during endochondral bone formation. J Cell Sci 113: 59-69.

Cramer, T., E. Schipanit, R.S. Johnson, B. Swoboda, D. Pfander (2004) Expression of VEGF isoforms by epiphyseal chondrocytes during low-oxygen is HIF-1 alpha dependent. Osteoarthritis Cartilage 12: 433-439.

Damron, T.A., J.A. Spadaro, B. Margulies, et al. (2000) Dose response of amifostine in protection of growth plate function from irradiation effects. Int J Cancer 90: 73-79.

Damron, T.A., B.S. Margulies, J.A. Strauss, et al. (2003) Sequential histomorphometric analysis of the growth plate following irradiation with and without radioprotection. J Bone Joint Surg Am 85-A: 1302-1313.

Damron, T.A., J.A. Spadaro, J.A. Horton, et al. (2004a) Novel radioprotectant drugs for sparing radiation-induced damage to the physis. Int J Radiat Biol 80: 217-228.

Damron, T.A., S. Mathur, J.A. Horton, et al. (2004b) Temporal changes in PTHrP, Bcl-2, Bax, caspase, TGF-beta, and FGF-2 expression following growth plate irradiation with or without radioprotectant. J Histochem Cytochem 52: 157-167.

Damron, T.A., J.A. Horton, A. Naqvi, et al. (2006) Combination radioprotectors maintain proliferation better than single agents by decreasing early parathyroid hormone-related protein changes after growth plate irradiation. Radiat Res 165: 350-358.

Damron, T.A., J.A. Horton, M.R. Pritchard, et al. (2008) Histomorphometric evidence of growth plate recovery potential after fractionated radiotherapy: an in vivo model. Radiat Res 170: 284-291.
Damron, R.A., M. Zhang, M.R. Pritchard, et al. (2009) Microarray cluster analysis of irradiated growth plate zones following laser microdissection. Int J Radiat Oncol Biol Phys 74: 949-956.

Erler, J.T., K.L. Bennewith, M. Nicolau, et al. (2006) Lysyl oxidase is essential for hypoxiainduced metastasis. Nature 440: 1222-1226.

Gille, H., J. Kowalski, B. Li, J. LeCouter, B. Moffat, T.F. Zioncheck, N. Pelletier, N. Ferrara (2001) Analysis of biological effects and signaling properties of Flt-1 (VEGFR-1) and KDR (VEGFR-2). J Biol Chem 276: 3222 3230 .

Goldwein, J.W. (1991) Effects of radiation therapy on skeletal growth in childhood. Clin Orthop Relat Res 262: 101-107.

Grewal, T.S., P.G. Genever, A.C. Brabbs, M Birch, T.M. Skerry (2000) Best5: a novel interferon-inducible gene expressed during bone formation. FASEB J 14: 523-531.

Hall, A.P., F.R. Westwood, P.F. Wadsworth (2006) Review of the effects of anti-angiogenic compounds on the epiphyseal growth plate. Toxicol Pathol 34: 131-147.

-Hardingham, T.E., R.A. Oldershaw, S.R. Tew (2006) Cartilage, SOX9 and Notch signals in chondrogenesis. J Anat 209: 469-480.

Horton, J.A., B.S. Margulies, J.A. Strauss, et al. (2006) Restoration of growth plate function following radiotherapy is driven by increased proliferative and synthetic activity of expansions of chondrocytic clones. J Orthop Res 24: 1945-1956.

Kanbe, K., K. Takagishi, Q. Chen (2002) Stimulation of matrix metalloprotease 3 release from human chondrocytes by the interaction of stromal cell-derived factor 1 and CXC chemokine receptor 4. Arthritis Rheum 46: 130-137.

Kitten, A.M., S.A. Harvey, N. Criscimagna, M. Asher, J.C. Lee, M.S. Olson (1997) Osteogenic protein-1 downregulates endothelin A receptors in primary rat osteoblats. Am J Physiol 272: E967-E975.

Koedam, J.A., J.J. Smink, S.C. van Buul-Offers (2002) Glucocorticoids inhibit vascular endothelial growth factor expression in growth plate chondrocytes. Mol Cell Endocrinol 197: 35-44.

Margulies, B., H. Morgan, M. Allen, et al. (2003) Transiently increased bone density after irradiation and the radioprotectant drug amifostine in a rat model. Am J Clin Oncol 26: e106-e114

Margulies, B.S., J.A. Horton, Y. Wang, et al. (2006) Effects of radiation therapy on chondrocytes in vitro. Calcif Tissue Int 78: $302-$ 313.
Monk, P.N., A.M. Scola, P. Madala, D.P. Fairlie (2007) Function, structure and therapeutic potential of complement C5a receptors. Br J Pharmacol 152: 429-448.

Motomura, H., H. Niimi, K. Sugimori, T. Ohtsuka, T. Kimura, I. Kitajima (2007) Gas6, a new regulator of chondrogenic differentiation from mesenchymal cells. Biochem Biophys Res Commun 357: 997-1003.

Ortega, N., D. Behonick, D. Stickens, Z. Werb (2003) How proteases regulate bone morphogensis. Ann NY Acad Sci 995: 109-116.

Robertson, W.W., Jr., M.S. Butler, G.J. D’Angio, et al. (1991) Leg length discrepancy following irradiation for childhood tumors. J Pediatr Orthop 11: 284-287.

-spadaro, J.A., M.T. Baesl, A.C. Conta, et al. (2003) Effects of irradiation on the appositional and longitudinal growth of the tibia and fibula of the rat with and without radioprotectant. J Pediatr Orthop 23: 35-40.

-Spadaro, J.A., J.A. Horton, B.S. Margulies, et al. (2005) Radioprotectant combinations spare radiation-induced damage to the physis more than fractionation alone. Int J Radiat Biol 81: 759-765.

-Tamurian, R.M., T.A. Damron, J.A. Spadaro (1999) Sparing radiation-induced damage to the physis by radioprotectant drugs: laboratory analysis in a rat model. J Orthop Res 17: 286-292.

Voet, D., J. Voet, C. Pratt (2002) Fundamentals of Biochemistry, rev ed. New Jersey, Wiley, pp 650-654.

Wang, Y., M. Zhang, F.A. Middleton, et al. (2007) Connective tissue growth factor and insulinlike growth factor 2 show upregulation in early growth plate radiorecovery response following irradiation. Cells Tissues Organs 186: $192-203$

Yui, S., Y. Nakatani, M. Hunter, W.J. Chazin, M. Yamazaki (2002) Implication of extracellular zinc exclusion by recombinant human calprotectin (MRP8 and MRP14) from target cells in it apoptosis-inducing activity. Mediators Inflamm 11: 165-172.

Zhang, M., Y. Wang, F.A. Middleton, et al. (2007) Growth plate zonal microarray analysis shows upregulation of extracellular matrix genes and downregulation of metalloproteinases and cathepsins following irradiation. Calcif Tissue Int 81: 26-38.

Zhang, M., M.R. Pritchard, F.A. Middleton, et al. (2008) Microarray analysis of perichondral and reserve growth plate zones identifies differential gene expressions and signal pathways. Bone 43: 511-520. DOI: 10.1016/j. bone.2008.04.021 